\title{
Risk factors for ischemic stroke in patients with non-valvular atrial fibrillation and therapeutic international normalized ratio range
}

\author{
Paweł Wańkowicz, Przemysław Nowacki, Monika Gołąb-Janowska
}

Department of Neurology, Pomeranian Medical University, Szczecin, Poland

Submitted: 15 May 2018

Accepted: 4 July 2018

Arch Med Sci 2019; 15 (5): 1217-1222

DOI: https://doi.org/10.5114/aoms.2018.77546

Copyright @ 2018 Termedia \& Banach

\section{Abstract}

Introduction: Atrial fibrillation (AF) is the most common cause of ischemic stroke (IS). Atrial fibrillation patients are recommended to use oral anticoagulants (OACS) as part of prevention against IS. However, despite having a therapeutic intensity of OAC therapy, IS can still occur in such patients. The aim of our study was to examine the configuration of IS risk factors in patients with non-valvular atrial fibrillation (NVAF) and within the therapeutic INR range (TINR).

Material and methods: Our retrospective study involved 1835 patients with a recent IS. The experimental group consisted of 154 patients with acute IS, NVAF and TINR. The control group consisted of 1681 patients with acute IS but without AF.

Results: Patients with IS, NVAF and TINR were significantly older and more often female than patients with IS without NVAF $(p<0.001$ and $p<0.001$, respectively). In these patients, diabetes mellitus, dyslipidemia, hypertension, coronary heart disease, smoking and previous IS were significantly more frequent than in the patients with IS without NVAF $(p=0.036, p=$ $0.002, p<0.001, p<0.001, p<0.001, p=0.003$ ). Based on a univariable and multivariable logistic regression model, we found that in the group of patients who suffered a stroke despite TINR compared to patients with IS without $A F$ there were more smokers $(O R=20.337$; $O R=147.589)$ and patients with previous stroke $(O R=6.556 ; O R=11.094)$, hypertension $(O R=$ $3.75 ; \mathrm{OR}=2.75)$ and dyslipidemia $(\mathrm{OR}=2.318 ; \mathrm{OR}=2.294)$.

Conclusions: The group of patients with NVAF and TINR is significantly more burdened by other independent common risk factors for stroke.

Key words: atrial fibrillation, ischemic stroke, therapeutic INR.

\section{Introduction}

Atrial fibrillation (AF) is the most common type of arrhythmia. Patients suffering from this condition may develop neurological deficiencies from an embolism or from hemodynamic disorders associated with the heart rhythm itself. AF predisposes to an increased risk of ischemic stroke (IS) by means of transfer of embolic material from the left atrium of the heart to the cerebral vessels [1]. In view of the above, AF patients are recommended to use oral anticoagulants (OACs) as part of secondary prevention against IS, such as vitamin $\mathrm{K}$ antagonists and new oral anticoagulants (NOACs). Anticoagulation reduced the risk of stroke from

\author{
Corresponding author: \\ Paweł Wańkowicz MD, PhD \\ Department of Neurology \\ Pomeranian Medical \\ University \\ 1 Unii Lubelskiej St \\ 71-252 Szczecin, Poland \\ Phone: +48 515079083 , \\ +48914253251 \\ E-mail: pawelwankowicz@ \\ gmail.com
}


$12 \%$ to $4 \%$ per year $[2,3]$. An interesting phenomenon and an important therapeutic problem is the occurrence of IS in patients with non-valvular atrial fibrillation (NVAF) and in the therapeutic INR range (TINR). With little information available in available literature regarding this problem, the aim of this study was to examine the configuration of IS risk factors in patients with NVAF and TINR.

\section{Material and methods}

Our retrospective study involved 1835 patients with a recent IS, hospitalized at the Department of Neurology, Pomeranian Medical University in Szczecin between January 2011 and December 2014. Patients were divided into two groups. The experimental group consisted of 154 patients (100 women and 54 men) with acute IS, NVAF and TINR. The control group consisted of 1681 patients (855 women and 826 men) with acute IS but without concurrent AF.

Atrial fibrillation was diagnosed based on information from a medical history or electrocardiographic results on admission or during hospitalization. Valvular heart diseases which were used as exclusion criterion from this study were identified based on the patient's history or echocardiographic tests during hospitalization. International normalized ratio was established at admission for each patient who received warfarin or acenocoumarol. In accordance with European guidelines for pharmacotherapy of AF, the recommended INR is from 2.0 to 3.0 for patients with NVAF [4]. Ischemic stroke was defined as sudden onset of a non-convulsive, focal neurological deficit persisting for more than $24 \mathrm{~h}$ by means of brain imaging (computed tomography (CT) and/or magnetic resonance imaging (MRI)). Each type of ischemic stroke, both cardioembolic and non-cardioembolic, including lacunar, atherothrombotic and various types, were included in the study. We collected data on the most common risk factors for IS from all patients. Written informed consent was obtained from each patient enrolled in the study. The Pomeranian Medical University Ethics Committee approved the study protocol, which conformed to the ethical guidelines of the Declaration of Helsinki.

Definitions for risk factors:

1. Atrial fibrillation was defined as the absence of $P$ waves in ECG, with the isoelectric line being replaced by irregular high-frequency oscillations (f waves), and wholly irregular ventricular response; this was based on ECG results during admission or in previous medical reports [5].

2. Diabetes mellitus type 2 was determined by the level of fasting blood glucose after a minimum of 2 test results $\geq 126 \mathrm{mg} / \mathrm{dl}$ or glucose level $\geq 200 \mathrm{mg} / \mathrm{dl}$ measured at any time during the day. An alternative criterion for diagnosing diabetes was a positive result of a glucose tolerance test (blood glucose $\geq 200 \mathrm{mg} / \mathrm{dl} 2 \mathrm{~h}$ after oral administration of $75 \mathrm{mg}$ of glucose) [6].

3. Dyslipidemia (serum cholesterol concentration $>190$ mg/dl, low-density lipoprotein (LDL) cholesterol $>115 \mathrm{mg} / \mathrm{dl}$, serum triglyceride concentration > $150 \mathrm{mg} / \mathrm{dl}$ and high-density lipoprotein (HDL) cholesterol $<40 \mathrm{mg} / \mathrm{dl}$ in males and $<45 \mathrm{mg} / \mathrm{dl}$ in females) [7].

4. Hypertension was diagnosed when in repeated tests the systolic blood pressure (SBP) was $\geq 140 \mathrm{~mm} \mathrm{Hg}$ and/or the diastolic blood pressure (DBP) was $\geq 90 \mathrm{~mm} \mathrm{Hg}$ [8].

5. Coronary heart disease was determined by previous history of angina pectoris, myocardial infarction, coronary artery bypass graft surgery or percutaneous transluminal coronary angioplasty.

6. Internal carotid artery hemodynamically significant stenosis was defined, with variation from study to study, ranging in degrees of stenosis from $50 \%$ to $70 \%$ and greater $[9,10]$.

7. Peripheral arterial disease includes previous history of intermittent claudication, arterial thrombosis, and percutaneous or surgical intervention in the thoracic, abdominal aorta, or lower extremity vessels.

8. Cigarette smoking was defined as current smoking of any number of cigarettes.

9. Previous stroke was considered to be present if the medical charts included a stroke diagnosis or if the individual, a caregiver, or a relative reported the diagnosis and it was found to be credible based on supporting information from medical charts.

\section{Statistical analysis}

The statistical null hypothesis was: there are no differences in risk factors between IS patients with NVAF with TINR and IS patients without AF. The alternative hypothesis was: there are significant differences in risk factors between IS patients with NVAF and TINR and IS patients without $A F$

In order to compare characteristics of NVAF and TINR stroke patients with patients without AF, the Mann-Whitney $U$ test for quantitative data was used. For qualitative data, Fisher's exact test was used. Multiple logistic regression was calculated for assessing the odds ratio of independent risk factors for stroke in IS patients with AF and TINR compared to patients without AF $(p<0.05$ was considered to indicate statistical significance). All calculations were performed in R statistical environment ( $R$ Version 3.4.4 2018-03-15). 


\section{Results}

Comparison of risk factors for ischemic stroke in patients with non-valvular atrial fibrillation and therapeutic INR range and patients with ischemic stroke without concurrent atrial fibrillation

A case comparison is presented in Table I. Older age, female gender, diabetes mellitus, dyslipidemia, hypertension, coronary heart disease, cigarette smoking and history of ischemic stroke were significantly more frequent in patients with concurrent NVAF and TINR than in patients without AF $(p<$ $0.001, p<0.001, p=0.036, p=0.002, p<0.001, p<$ $0.001, p<0.001, p=0.003)$. Interestingly, significant ICA stenosis/occlusion was only slightly more frequent in the group of patients without $\operatorname{AF}(p=0.03)$.

\section{Analysis of risk factors of ischemic stroke in patients with non-valvular atrial fibrillation who suffered a stroke despite TINR compared to patients without AF}

Based on a univariable and multivariable logistic regression model, we found that in the group of patients who suffered a stroke despite TINR compared to patients with IS without AF there were more smokers $(O R=20.337 ; O R=147.589)$ and patients with previous stroke $(\mathrm{OR}=6.556$; $\mathrm{OR}=$ 11.094), hypertension $(O R=3.75 ; O R=2.75)$ and dyslipidemia $(O R=2.318 ; O R=2.294)$ (Table II).

\section{Discussion}

In this study we confirmed that in addition to the embolic factor associated with AF, patients with TINR were significantly more likely to have other risk factors, including those conducive to thrombotic and thromboembolic mechanisms. Therefore, in the above-mentioned group of patients the risk of developing IS was high even when the INR values remained in the therapeutic range. This seems to confirm the results of a study conducted by Evans et al. [11] in which a group of patients with IS and concurrent AF were subjected to a 2-year follow-up. The patients had been treated with warfarin. It was observed that the frequency of lacunar stroke recurrence was higher than that of cardioembolic stroke. According to our analysis, patients with IS, NVAF and TINR were significantly older and mostly female $(p<0.001$ and $p<0.001$, respectively). In these patients, type 2 diabetes, dyslipidemia, hypertension, coronary heart disease, smoking and previous IS were significantly more frequent than in the patients with IS without AF ( $p=0.036, p=0.002, p$ $<0.001, p<0.001, p<0.001, p=0.003)$. TINR in this group of patients did not reduce the risk of IS. Based on a univariable and multivariable logistic regression model, we found that in the group of patients who suffered a stroke despite TINR compared to patients with IS without AF there were more smokers $(O R=20.337 ; O R=147.589)$ and patients with previous stroke $(O R=6.556$; $O R=$ 11.094), hypertension $(O R=3.75 ; O R=2.75)$ and dyslipidemia (OR = 2.318; OR = 2.294). It should be emphasized that among patients with ICA, significant stenosis/occlusion only slightly predominated in the group without $A F$, which means that in many patients with AF, ICA and stenosis/ occlusion coexist as an important risk factor for

Table I. Characteristics of patients with ischemic stroke and non-valvular atrial fibrillation as well as therapeutic INR and patients with ischemic stroke without atrial fibrillation

\begin{tabular}{|c|c|c|c|}
\hline Parameter & TINR $(n=154)$ & $\mathrm{AF}-(n=1681)$ & $P$-value \\
\hline Age & $77.6 \pm 10.4$ & $69.8 \pm 12.5$ & $<0.001$ \\
\hline Female, $n(\%)$ & $100(64.9)$ & $855(50.9)$ & $<0.001$ \\
\hline \multicolumn{4}{|l|}{ Comorbidities, $n(\%)$ : } \\
\hline Diabetes mellitus & $59(38.3)$ & $514(30.6)$ & 0.036 \\
\hline Dyslipidemia & $81(52.6)$ & $680(40.5)$ & 0.002 \\
\hline Hypertension & $143(92.9)$ & $1243(73.9)$ & $<0.001$ \\
\hline \multicolumn{4}{|l|}{ ICA significant, $n(\%)$ : } \\
\hline Stenosis/occlusion & $13(8.1)$ & $250(14.8)$ & 0.03 \\
\hline Coronary heart disease & $92(59.7)$ & 705 (41.9) & $<0.001$ \\
\hline Peripheral arterial disease & $14(9.1)$ & $162(9.6)$ & 0.86 \\
\hline Smoking & $101(65.6)$ & $883(52.5)$ & $<0.001$ \\
\hline Previous stroke, $n$ (\%) & $92(59.7)$ & $733(43.7)$ & 0.003 \\
\hline
\end{tabular}

ICA - internal carotid artery. 
Table II. Analysis of risk factors of ischemic stroke in patients with non-valvular atrial fibrillation, who suffered a stroke despite TINR compared to patients without AF (multivariate logistic regression)

\begin{tabular}{|c|c|c|c|c|c|c|}
\hline \multirow[t]{2}{*}{ Factors } & \multicolumn{3}{|c|}{ Univariable logistic regression models } & \multicolumn{3}{|c|}{ Multivariable logistic regression model ${ }^{*}$} \\
\hline & OR & $\mathrm{Cl}$ & $P$-value & OR & $\mathrm{Cl}$ & $P$-value \\
\hline Age & 1.058 & $1.041-1.075$ & $<0.01$ & 1.113 & $1.084-1.145$ & $<0.01$ \\
\hline \multicolumn{7}{|c|}{ Hypertension: } \\
\hline Yes & 3.75 & $2.1-7.432$ & $<0.01$ & 2.751 & $1.263-6.65$ & 0.016 \\
\hline No & 1.0 & - & - & 1.0 & - & - \\
\hline \multicolumn{7}{|l|}{ Smoking: } \\
\hline Yes & 20.337 & $13.618-31.143$ & $<0.01$ & 147.589 & $74.213-316.588$ & $<0.01$ \\
\hline No & 1.0 & - & - & 1.0 & - & - \\
\hline \multicolumn{7}{|c|}{ Previous stroke: } \\
\hline Yes & 6.556 & $4.621-9.359$ & $<0.01$ & 11.094 & $6.355-20.096$ & $<0.01$ \\
\hline No & 1.0 & - & - & 1.0 & - & - \\
\hline \multicolumn{7}{|c|}{ Dyslipidemia: } \\
\hline Yes & 2.318 & $1.649-3.278$ & $<0.01$ & 2.294 & $1.369-3.896$ & $<0.01$ \\
\hline No & 1.0 & - & - & 1.0 & - & - \\
\hline \multicolumn{7}{|c|}{ ICA significant stenosis/occlusion: } \\
\hline Yes & 0.491 & $0.261-0.85$ & 0.017 & 0.418 & $0.188-0.863$ & 0.024 \\
\hline No & 1.0 & - & - & 1.0 & - & - \\
\hline \multicolumn{7}{|l|}{ Gender: } \\
\hline Female & 1.0 & - & - & 1.0 & - & - \\
\hline Male & 0.583 & $0.408-0.824$ & $<0.01$ & 0.366 & $0.206-0.64$ & $<0.01$ \\
\hline \multicolumn{7}{|c|}{ Diabetes mellitus: } \\
\hline Yes & 1.306 & $0.917-1.845$ & 0.134 & 1.081 & $0.641-1.809$ & 0.768 \\
\hline No & 1.0 & - & - & 1.0 & - & - \\
\hline \multicolumn{7}{|c|}{ Coronary heart disease: } \\
\hline Yes & 1.945 & $1.383-2.751$ & $<0.01$ & 1.864 & $1.112-3.164$ & 0.019 \\
\hline No & 1.0 & - & - & 1.0 & - & - \\
\hline
\end{tabular}

${ }^{*}$ Full adjusted model; Independent variables: age; hypertension (yes/no); smoking (yes/no); previous stroke (yes/no); dyslipidemia (yes/ no); ICA significant stenosis/occlusion; sex (F/M); diabetes mellitus (yes/no); coronary heart disease (yes/no). ICA - internal carotid artery.

stroke which requires different treatment than AF. On one hand, a significant symptomatic stenosis of the artery favors small embolisms already in the extra-cranial section of the artery, and on the other hand it can be a direct cause of a stroke in the hypoperfusion mechanism associated with hemodynamic disturbances resulting from AF. It may also lead to a stroke independently in a thromboembolic mechanism (arterio-arterial). An attempt to combine pharmacological management in secondary stroke prevention in patients with AF (recommended anticoagulant) and that used for ICA stenosis (antiaggregant) would significantly increase the risk of intracranial hemorrhagic complications.
In 2010, the results of the INTERSTROKE survey were announced. In this study, the authors observed that hypertension, smoking, obesity, diet and lack of physical activity are the most important modifiable risk factors for stroke with both ischemic and hemorrhagic etiology. They cause that the risk of stroke is $80 \%$. Including the next five factors - diabetes, alcohol abuse, psychosocial factors, heart disease and apolipoprotein B to A1 ratio - risk of stroke increases to $90 \%$. It is also interesting to compare the risk factors assessed in the INTERSTROKE and INTERHEART trials. The risk factors responsible for $90 \%$ of the risk of myocardial infarction and stroke are the same. The differ- 
ences concern only their impact strength. While in stroke the most important risk factor is hypertension, in the case of myocardial infarction it is lipid disorders. Patients with stroke are less likely to smoke, have less alcohol abuse and rarely have diabetes [12, 13].

Hypertension is not only one of the most frequent causes of first stroke, but also increases the risk of recurrent stroke in patients who have had this episode in the past, and the risk of morbidity and mortality from cardiovascular causes [14]. Treatment of hypertension significantly reduces the risk of vascular death and general mortality, and the reduction of risk depends on the degree of pressure reduction. The results of the most important prospective studies on antihypertensive therapy indicate that reduction of systolic blood pressure by $10-12 \mathrm{~mm} \mathrm{Hg}$ and diastolic pressure by $5-6 \mathrm{~mm} \mathrm{Hg}$ allows the number of strokes to be reduced by $38 \%$ [15]. In addition, obtaining a diastolic pressure lower than or equal to $80 \mathrm{~mm} \mathrm{Hg}$ as a result of treatment causes a $43 \%$ reduction in the incidence of stroke in relation to people with a pressure less than or equal to $90 \mathrm{~mm} \mathrm{Hg}$ [16]. Perhaps this is the answer to the question of the optimal value to which blood pressure should be lowered to reduce the risk of stroke.

Diabetes is combined with both large and small vessel disease, and the relative risk of stroke in patients with diabetes is estimated from 1.8 to 6.0 , with that of the male and female population being $4.1 \%$ and $5.8 \%$, respectively, on average [17]. The risk depends on the type and severity of the disease [18]. The risk of stroke in diabetic patients increases when the patient with diabetes also has other risk factors for stroke [19].

Smoking is a major cause of disability and deaths, being responsible for $6.3 \%$ of all illnesses [20]. Based on many multicenter studies, smoking has been confirmed as an important and independent risk factor for IS. Smokers are 2 to 4 times more likely to have ischemic strokes than non-smokers, regardless of the presence of other risk factors [21, 22].

Dyslipidemia is the main risk factor for coronary heart disease; its role in the pathogenesis of ischemic stroke, initially unclear, is now more and more appreciated. Dyslipidemia and cigarette smoking are responsible for insulin resistance and are modifiable risk factors for vasculopathy. Insulin resistance causes inhibition of lipogenesis, lipolysis intensification, and thus an increased concentration of free fatty acids and triglycerides in the blood [23]. The risk of the first IS is reduced by $21 \%$ for each LDL-C reduction by $1 \mathrm{mmol} / \mathrm{l}$. This effect is similar in men and women $[24,25]$. Beneficial effects persist over long-term observation. In the United Kingdom Prospective Diabetes Study in people with newly diagnosed type 2 diabetes, an increase in $\mathrm{HDL}$ by $3.86 \mathrm{mg} / \mathrm{dl}$ reduced the risk of stroke by $15 \%$ [26].

This study had several of limitations inherent to its retrospective cohort methodology. This was a single-center study, with a limited number of TINR patients. We selected only the most common stroke risk factors. Further prospective studies with a larger sample size are needed.

In conclusion, as evidenced by our results, in patients with NVAF and co-existing thromboembolic risk factors, warfarin treatment and INR index in the 2.0-3.0 range are insufficient to protect this group of patients against an IS. Increasing INR to more than 3, recommended by some authors, or replacing old generation anticoagulants with new generation oral anticoagulants also will not protect the patients with NVAF and thromboembolic concomitant factors against IS [27-30]. This is due to the fact that, apart from NVAF, numerous other risk factors require the use of an antiaggregate agent. The combination of an anticoagulant with an antiaggregant, due to the significant risk of intracerebral hemorrhage, has so far not been justified in the secondary prevention of IS. This seems to increase the role of statins in addition to the recognized anticoagulant role in secondary prevention of stroke in patients with NVAF. In light of the above data, it seems increasingly important to modify the lifestyle of patients, including dietary treatment, ceasing smoking and, if possible, increasing physical activity.

\section{Conflict of interest}

The authors declare no conflict of interest.

\section{References}

1. Fatkin D, Kelly RP, Feneley MP. Relations between left atrial appendage blood flow velocity, spontaneous echocardiographic contrast and thromboembolic risk in vivo. J Am Coll Cardiol 1994; 23: 961-9.

2. EAFT (European Atrial Fibrillation Trial) Study Group. Secondary prevention in non-rheumatic atrial fibrillation after transient ischaemic attack or minor stroke. Lancet 1993; 342: 1255-62.

3. Verheugt FW, Granger CB. Oral anticoagulants for stroke prevention in atrial fibrillation: current status, special situations, and unmet needs. Lancet 2015; 386: 303-10.

4. The Task Force for the Management of Atrial Fibrillation of the European Society of Cardiology (ESC). Eur Heart J 2010; 31: 2369-29.

5. Jaroszyński A, Jaroszyńska A, Dąbrowski W, et al. Factors influencing $P$ terminal force in lead V1 of the ECG in hemodialysis patients. Arch Med Sci 2018; 14: 257-64.

6. Tahrani AA, Bailey CJ, Del Prato S. Management of type 2 diabetes: new and future developments in treatment. Lancet 2011; 378: 182-97.

7. Grundy SM, Cleeman JI, Merz NB, et al. Implications of recent clinical trials for the National Cholesterol Education Program Adult Treatment Panel III guidelines. Arterioscler Thromb Vasc Biol 2004; 8: e149-61. 
8. Mancia G, De Backer G, Dominiczak A, et al. Management of Arterial Hypertension of the European Society of Hypertension/European Society of Cardiology. 2007 Guidelines for the Management of Arterial Hypertension: The Task Force for the Management of Arterial Hypertension of the European Society of Hypertension $(\mathrm{ESH})$ and of the European Society of Cardiology (ESC). J Hypertens 2007; 25: 1105-87.

9. Inzitari D, Eliasziw M, Gates P, et al. The causes and risk of stroke in patients with asymptomatic internal-carotid-artery stenosis. North American Symptomatic Carotid Endarterectomy Trial Collaborators. N Engl J Med 2000; 342: 1693-700.

10. Halliday A, Harrison M, Hayter E, et al. 10-year stroke prevention after successful carotid endarterectomy for asymptomatic stenosis (ACST-1): a multicentre randomised trial. Lancet 2010; 376: 1074-84.

11. Evans A, Perez I, Yu G, Kalra L. Should stroke subtype influence anticoagulation decisions to prevent recurrence in stroke patients with atrial fibrillation? Stroke 2001; 32: 2828-32

12. O’Donnell MJ, Xavier D, Liu L, et al. Risk factors for ischemic and intracerebral haemorrhagic stroke in 22 countries (the INTERSTROKE study): a case - control study. Lancet 2010; 376: 112-23.

13. Yusuf S, Hawken S, Ounpun S, et al. Effect of potentially modificable risk factors associated with myocardial infarction in 52 countries (the INTERHEART study): case-control study. Lancet 2004; 364: 937-52.

14. 2007 Guidelines for the Management of Arterial Hypertension. The Task Force for the Management of Arterial Hypertension of the European Society of Hypertension $(\mathrm{ESH})$ and of the European Society of Cardiology (ESC). J Hypertens 2007; 25: 1105-87.

15. MacMahon S. Blood pressure and the prevention of stroke. J Hypertens 1996; 14: S39-46.

16. Hansson L, Zanchetti A, Carruthers SG, et al. Effects of intensive blood-pressure lowering and low-dose aspirin in patients with hypertension: principal results of the Hypertension Optimal Treatment (HOT) randomised trial. HOT Study Group. Lancet 1998; 351: 1755-62.

17. Kissela BM, Khoury J, Kleindorfer D, et al. Epidemiology of ischemic stroke in patients with diabetes: the greater Cincinnati/Northern Kentucky Stroke Study. Diab Care 2005; 28: 355-9.

18. Booth GL, Kapral MK, Fung K, Tu JV. Relation between age and cardiovascular disease in men and women with diabetes compared with non-diabetic people: a population-based retrospective cohort study. Lancet 2006; 368: 29-35.

19. Lindsberg PJ, Roine RO. Hyperglycemia in acute stroke. Stroke 2004; 35: 363-4.

20. Lim SS, Vos T, Flaxman AD, et al. A comparative risk assessment of burden of disease and injury attributable to 67 risk factors and risk factor clusters in 21 regions, 1990-2010: a systematic analysis for the Global Burden of Disease Study 2010. Lancet 2012; 380: 2224-60.

21. Mannami T, Iso H, Baba S, et al. Cigarette smoking and risk of stroke and its subtypes among middle-aged Japanese men and women: the JPHC Study Cohort I. Stroke 2004; 35: 1248-53.

22. Gill JS, Shipley MJ, Tsementzis SA, et al. Cigarette smoking. A risk factor for hemorrhagic and nonhemorrhagic stroke. Arch Int Med 1989; 149: 2053-7.

23. Unger RH. Lipid overload and overflow: metabolic trauma and the metabolic syndrome. Trends Endocrinol Metab 2003; 14: 398-403.
24. Cholesterol Treatment Trialists' (CTT) Collaboration, Baigent $C$, Blackwell L, et al. Efficacy and safety of more intensive lowering of LDL cholesterol: a meta-analysis of data from 170,000 participants in 26 randomised trials. Lancet 2010; 376: 1670-81.

25. Heart Protection Study Collaborative Group. Effects on 11-year mortality and morbidity of lowering LDL choles terol with simvastatin for about 5 years in 20,536 highrisk individuals: a randomised controlled trial. Lancet 2011; 378: 2013-20.

26. UK Prospective Diabetes Study Group. Tight blood pres sure control and risk of macrovascular and microvas cular complications in type 2 diabetes. UKPDS 38. BMJ 1998; 317: 703-13.

27. Fuster V, Rydén LE, Cannom DS, et al. 2011 ACCF/AHA/ HRS focused updates incorporated into the ACC/AHA ESC 2006 guidelines for the management of patients with atrial brillation: a report of the American College of Cardiology Foundation/American Heart Association Task Force on practice guidelines. Circulation 2011; 123: e269-367.

28. Ruiz-Irastorza G, Khamashta MA, Hunt BJ, et al. Bleeding and recurrent thrombosis in definite antiphospholipid syndrome: analysis of a series of 66 patients treated with oral anticoagulation to a target international normalized ratio of 3.5. Arch Intern Med 2002; 162: 1164-9.

29. Ntaios G, Papavasileiou V, Diener HC, et al. Nonvitamin-K-antagonist oral anticoagulants in patients with atrial fibrillation and previous stroke or transient ischemic attack: a systematic review and meta-analysis of randomized controlled trials. Stroke 2012; 43: 3298-304.

30. Albertsen IE, Rasmussen LH, Overvad TF, Graungaard T, Larsen TB, Lip GY. Risk of stroke or systemic embolism in atrial fibrillation patients treated with warfarin: a systematic review and meta-analysis. Stroke 2013; 44: 1329-36. 